\title{
Semiexclusive production of vector mesons in proton-proton collisions with electromagnetic dissociation of protons
}

\author{
Anna Cisek ${ }^{1, *}$, Wolfgang Schäfer ${ }^{2}$, and Antoni Szczurek ${ }^{1},{ }^{2}$ \\ ${ }^{1}$ Faculty of Mathematics and Natural Sciences, University of Rzeszów, ul. Pigonia 1, PL-35-310 \\ Rzeszów, Poland \\ ${ }^{2}$ Institute of Nuclear Physics, Polish Academy of Sciences, ul. Radzikowskiego 152, PL-31-342 \\ Kraków, Poland
}

\begin{abstract}
We discuss the semiexclusive production of vector mesons in protonproton collisions with electromagnetic dissociation of one of the protons. Several differential distribution in missing mass $\left(M_{X}\right)$, or single-particle variables related exclusively to the produced vector meson are calculated for pp centerof-mass energies 7 and $13 \mathrm{TeV}$. The cross sections and some differential distributions are compared to their counterparts for purely exclusive reaction $p p \rightarrow p V p$. For electromagnetic dissociation the important property is that the $p \gamma^{\star} \rightarrow X p$ transitions are given by the electromagnetic structure function of proton. In our calculations we use different parametrizations of the structure function and discuss how it is constrained by the data on virtual photoabsorption on a proton.
\end{abstract}

\section{Introduction}

Exclusive vector meson production, especially of heavier quarkonia like $J / \psi$ or $\Upsilon$ in $\gamma p \rightarrow$ $V p$ collisions is in principle subjected to pQCD methods. The energy dependence for the diffractive photoproduction of vector mesons was measured at the HERA accelerator. This dependence shows that for light vector mesons soft processes dominate and for the heavy vector mesons hard processes dominate. The energy dependence of the diffractive cross section is driven by the gluon distribution. In addition to purely elastic case $\gamma p \rightarrow V p$ also inelastic $\gamma^{\star} p \rightarrow V X$ processes with a large rapidity gap between the vector meson and the final state inelastic system $(\mathrm{X})$ were observed in experiments performed at HERA [1]. In recent years, the diffractive photoproduction of vector mesons in the $p p \rightarrow p V p$ process has been studied at the LHC $[2,3]$ for higher energy than those available at HERA. The first data show that in addition to exclusive production there is also semiexclusive production. For proton-proton collisions, two types of proton excitations are possible: diffractive and electromagnetic [4]. Our attention was focused rather on electromagnetic exctitations for which the results are presented here.

\footnotetext{
*e-mail: acisek@ur.edu.pl
} 


\section{Semiexclusive production with electromagnetic dissociation of protons}

The semiexclusive production of vector mesons in proton-proton collisions with electromagnetic dissociation of protons is illustrated in Fig. 1.
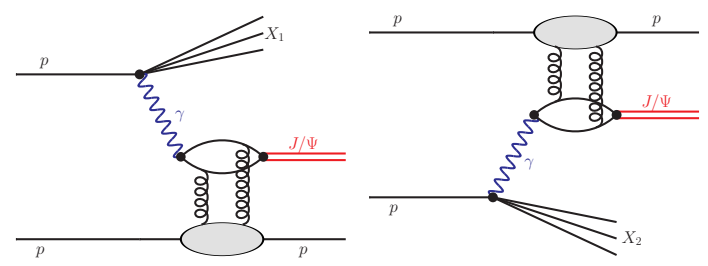

Figure 1. Schematic representation of the electromagnetic excitation of one (left panel) or second (right panel) proton.

The important property of these processes is that the $\gamma^{\star} p \rightarrow V X$ transition is given by the electromagnetic structure functions of the proton. The cross section for such processes can be written as:

$$
\frac{d \sigma(p p \rightarrow X V p ; s)}{d y d^{2} \boldsymbol{p} d M_{X}^{2}}=\int \frac{d^{2} \boldsymbol{q}}{\pi \boldsymbol{q}^{2}} \mathcal{F}_{\gamma / p}^{(\mathrm{in})}\left(z_{+}, \boldsymbol{q}^{2}\right) \frac{1}{\pi} \frac{d \sigma^{\gamma^{*} p \rightarrow V p}}{d t}\left(z_{+} s, t=-(\boldsymbol{q}-\boldsymbol{p})^{2}\right)+\left(z_{+} \leftrightarrow z_{-}\right) .
$$

Photons carry a longitudinal momentum fraction $z_{ \pm}=e^{ \pm y} \sqrt{\boldsymbol{p}^{2}+m_{V}^{2}} / \sqrt{s}$ and transverse momentum q.

The effective photon flux in dissociative events can be expressed through the structure function $F_{2}$ as:

$$
\mathcal{F}_{\gamma / p}^{(\text {inel })}\left(z, \boldsymbol{q}^{2}, M_{X}^{2}\right)=\frac{\alpha_{\mathrm{em}}}{\pi}(1-z) \theta\left(M_{X}^{2}-M_{\mathrm{thr}}^{2}\right) \frac{F_{2}\left(x_{B j}, Q^{2}\right)}{M_{X}^{2}+Q^{2}-m_{p}^{2}}\left[\frac{\boldsymbol{q}^{2}}{\boldsymbol{q}^{2}+z\left(M_{X}^{2}-m_{p}^{2}\right)+z^{2} m_{p}^{2}}\right]^{2},
$$

with

$$
Q^{2}=\frac{1}{1-z}\left[\boldsymbol{q}^{2}+z\left(M_{X}^{2}-m_{p}^{2}\right)+z^{2} m_{p}^{2}\right], x_{B j}=\frac{Q^{2}}{Q^{2}+M_{X}^{2}-m_{p}^{2}} .
$$

In practical calculation we used different parametrizations of electromagnetic structure functions of the proton [5-7].

\section{Results}

In this section we shall show some differential distributions associated with electromagnetic excitation of the final state proton. In our calculation we used different parametrizations of electromagnetic structure function $F_{2}$ of the proton.

In Fig. 2 we show the rapidity distribution for semiexclusive production of vector mesons for collision energy of $7 \mathrm{TeV}$ (upper plots) and $13 \mathrm{TeV}$ (lower plots). In the left panel we present results for $\phi$ meson, in the middle panel for $J / \psi$ meson and in the right panel for $\Upsilon$ meson. We show results for different parametrizations of the $F_{2}$ structure function. The blue solid lines are for Abramowicz-Levin-Levy-Maor (ALLM) [5], the red dash-dotted lines are for Fiore-Flachi-Jankovszky-Lengyel-Magas (FFJLM) [6], the green dashed lines are for Szczurek-Uleshchenko (SU) [6] fits to $F_{2}$ and the dotted lines are for VDM contribution 
alone. In Fig. 3 we show the transverse momentum distribution for semiexclusive production of vector mesons for energy $7 \mathrm{TeV}$ (upper plots) and $13 \mathrm{TeV}$ (lower plots). In the left panel we presents results for $\phi$ meson, in the middle panel for $J / \psi$ meson and in the right panel for $\Upsilon$ meson. W show results for different parametrizations of the $F_{2}$ structure function. The notation of lines are the same as in Fig. 2. The results presented in Fig. 2 and Fig. 3 are for missing mass $M_{X}<10 \mathrm{GeV}$. The FFJLM parametrization is reasonable only for $M_{X}<2 \mathrm{GeV}$ while the ALLM parametrization works well in a much broader range of missing masses $M_{X}$.

In Fig. 4 we show the sums of the two single-proton excitations. The presented results are for energy $7 \mathrm{TeV}$ and different cuts on missing mass. In the left panel we show results for $M_{X}<2 \mathrm{GeV}$, in the middle panel for $M_{X}<5 \mathrm{GeV}$ and in the right panel for $M_{X}<10 \mathrm{GeV}$. We show contribution of low-mass electromagnetic excitation with FFJLM (dotted line) and ALLM (dashed line) parametrizations of the $F_{2}$ structure functions. Similarly we show contributions of diffractive partonic (short-dashed line) and resonance (dash-dotted line) contributions. For comparison we show also contribution of purely exclusive process $p p \rightarrow p J / \psi p$ (top solid line) [8]. We predict that the contribution of low mass excitations gives camellike shapes with maxima at $y \approx \pm 4$. When higher (nonresonant) mass region is included the semiexclusive contribution grows considerably and the two separated maxima merge into one maximum at $y=0$.
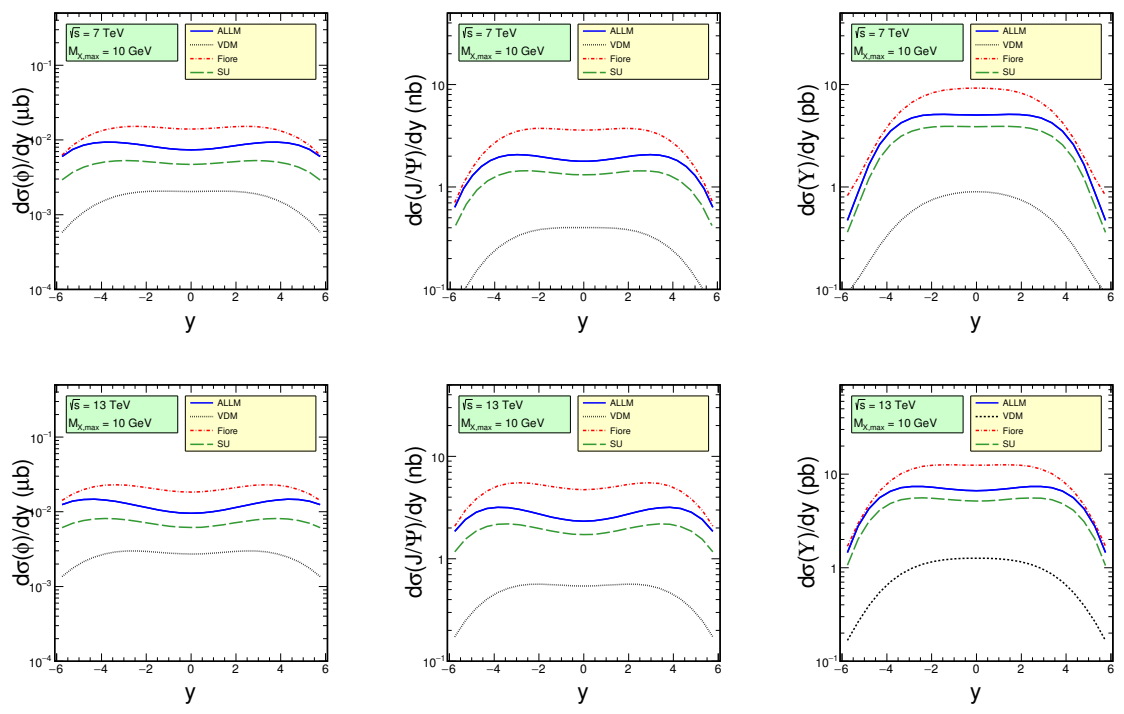

Figure 2. Rapidity distribution of vector mesons produced when one of the protons is excited due to photon exchange. Both contributions (excitations) are added together. 

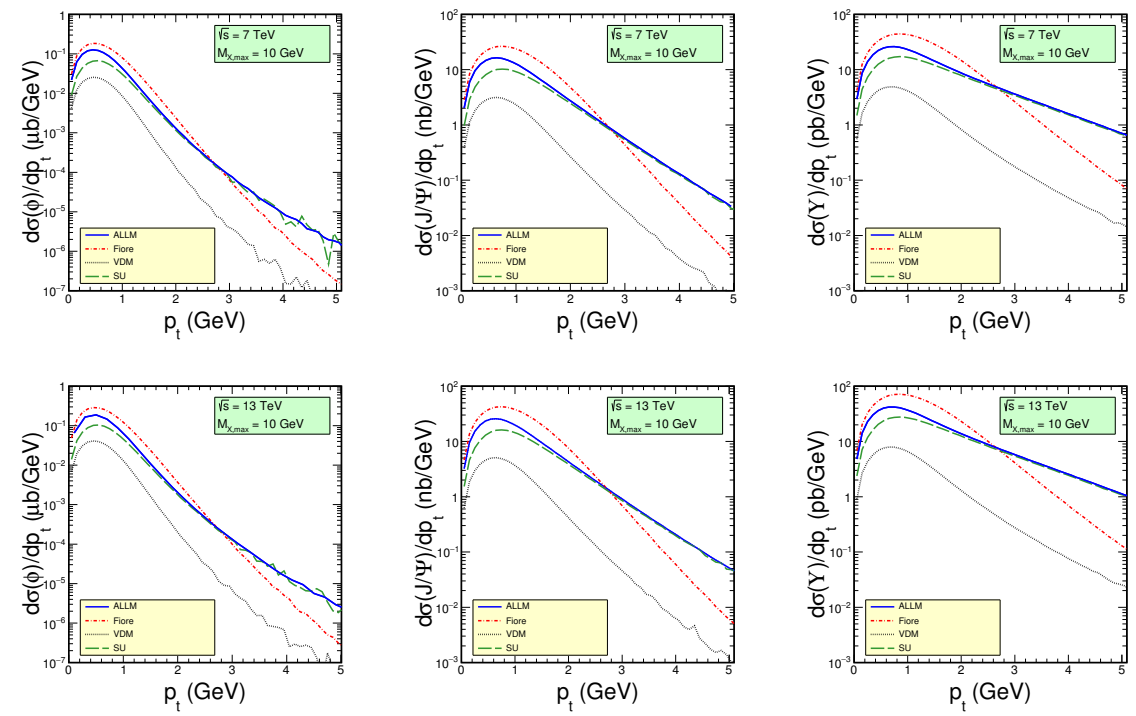

Figure 3. Transverse momentum distribution of vector mesons produced when only one of the protons is excited due to the photon exchange. Here both contributions are added together.
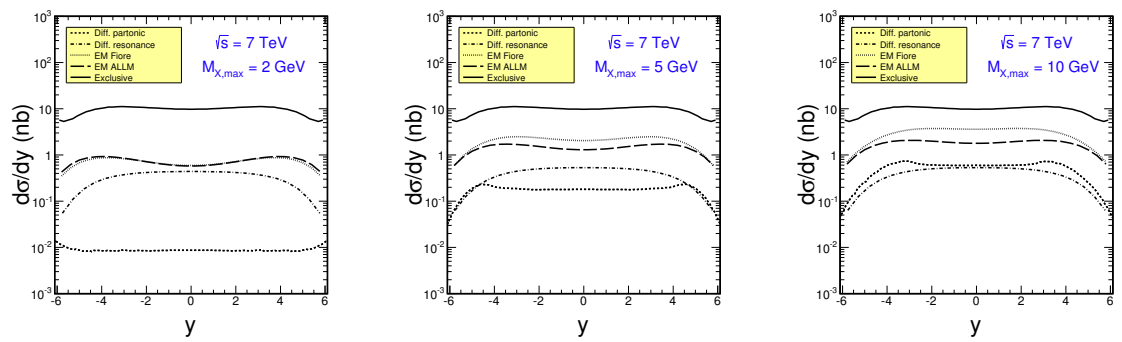

Figure 4. Rapidity distribution of $J / \psi$ meson produced when only one of the protons is excited due to photon or Pomeron exchange. Both contributions (first or second proton excitation) are added together. We also show a reference distribution for the $p p \rightarrow p V p$ exclusive process with parameters taken from [8].

\section{References}

[1] C. Alexa et al. [H1 Collaboration], Eur. Phys. J. C 73, 2466 (2013)

[2] R. Aaij et al. [LHCb Collaboration], J. Phys. G 40, 045001 (2013)

[3] R. Aaij et al. [LHCb Collaboration], J. High Energy Phys. 1509, 084 (201)

[4] A. Cisek, W. Schäfer and A. Szczurek, Phys. Lett. B 769, 176 (2017)

[5] H. Abramowicz, E. M. Levin, A. Levy and U. Maor, Phys. Lett. B 269, 465 (1991)

[6] R. Fiore, A. Flachi, L. L. Jankovszky, A. I. Lengyel and V. K. Magas, Phys. Rev. D 70, 054003 (2004)

[7] A. Szczurek and V. Uleshchenko, Eur. Phys. J. C 12, 663 (2000)

[8] A. Cisek, W. Schäfer and A. Szczurek, J. High Energy Phys. 1504, 159 (2015) 